\title{
Rapid identification of Legionella species by mass spectrometry
}

\author{
Claire Moliner, ${ }^{1}$ Christophe Ginevra, ${ }^{2,3,4,5}$ Sophie Jarraud, ${ }^{2,3,4,5}$ \\ Christophe Flaudrops, ${ }^{6}$ Marielle Bedotto, ${ }^{6}$ Carine Couderc, ${ }^{6}$ \\ Jerome Etienne $e^{2,3,4,5}$ and Pierre-Edouard Fournier ${ }^{1,6}$
}

\begin{abstract}
Correspondence
Pierre-Edouard Fournier pierre-edouard.fournier@ univmed.fr
\end{abstract}

Received 3 July 2009

Accepted 15 November 2009

\author{
${ }^{1}$ URMITE CNRS-IRD UMR 6236, Faculté de Médecine, 27 boulevard Jean Moulin, 13385 Marseille \\ Cedex 05, France \\ ${ }^{2}$ Université de Lyon, Lyon, France \\ ${ }^{3}$ INSERM, U851, 21 avenue Tony Garnier, 69007 Lyon, France \\ ${ }^{4}$ Université Lyon 1, IFR128, Lyon, France \\ ${ }^{5}$ Hospices Civils de Lyon, Faculté de Médecine Lyon Est Claude Bernard, Lyon, France \\ ${ }^{6}$ Fédération de Microbiologie Clinique, Hôpital de la Timone, 264 rue Saint-Pierre, 13385 Marseille \\ Cedex 05, France
}

\begin{abstract}
Legionella species are facultative, intracellular bacteria that infect macrophages and protozoa, with the latter acting as transmission vectors to humans. These fastidious bacteria mostly cause pulmonary tract infections and are routinely identified by various molecular methods, mainly PCR targeting the mip gene and sequencing, which are expensive and time-consuming. Recently, matrix-assisted laser desorption/ionization time-of-flight mass spectrometry (MALDI-TOF-MS) has emerged as a rapid and inexpensive method for identification of bacterial species. This study evaluated the use of MALDI-TOF-MS for rapid species and serogroup identification of 21 Legionella species recognized as human pathogens. To this end, a reference MS database was developed including 59 Legionella type strains, and a blind test was performed using 237 strains from various species. Two hundred and twenty-three of the 237 strains (94.1\%) were correctly identified at the species level, although ten (4.2\%) were identified with a score lower than 2.0. Fourteen strains $(5.9 \%)$ from eight species were misidentified at the species level, including seven $(3.0 \%)$ with a significant score, suggesting an intraspecific variability of protein profiles within some species. MALDI-TOF-MS was reproducible but could not identify Legionella strains at the serogroup level. When compared with mip gene sequencing, MALDI-TOF-MS exhibited a sensitivity of 99.2 and $89.9 \%$ for the identification of Legionella strains at the genus and species level, respectively. This study demonstrated that MALDI-TOF-MS is a reliable tool for the rapid identification of Legionella strains at the species level.
\end{abstract}

\section{INTRODUCTION}

Legionella species are fastidious, Gram-negative bacteria that belong to the Gammaproteobacteria. They are facultative, intracellular parasites infecting macrophages and protozoa, and are naturally associated with free-living amoebae, which may act as transmission vectors to humans. Legionella species mostly cause pulmonary tract infections. Legionella pneumophila is the most common pathogenic species for humans, and thus the most studied. Other proven pathogenic Legionella species include $L$. anisa, L. birminghamensis, L. bozemanae, L. cincinnatiensis,

Abbreviation: MALDI-TOF-MS, matrix-assisted laser desorption/ionization time-of-flight mass spectrometry.
L. dumoffii, L. feeleii, L. gormanii, L. hackeliae, L. jordanis, L. lansingensis, L. longbeachae, L. lytica, L. maceachernii, $L$. micdadei, L. oakridgensis, $L$. parisiensis, $L$. sainthelensi, $L$. tucsonensis and L. wadsworthii (Garrity et al., 2005).

Molecular methods have become major tools for the identification of fastidious bacteria. For Legionella species, several molecular tools have been described, including PCR targeting the mip gene (Ratcliff et al., 1998). This gene, currently the mostly commonly used PCR target, encodes an immunophilin of the FK506-binding protein class (Hacker \& Fischer, 1993). The mip gene exhibits 3-31\% nucleotide sequence variation among Legionella species and appears to be genetically stable (Ratcliff et al., 1998). Other discriminatory molecular methods include PCR 
Table 1. Legionella type strains used in this study

Strains shaded in grey are those for which spectra are available in the Bruker database.

\begin{tabular}{|c|c|c|c|c|}
\hline Species & Subspecies & Strain name & Serogroup & Culture collection no.* \\
\hline L. pneumophila & pneumophila & Philadelphia-1 & 1 & ATCC 33152 \\
\hline L. pneumophila & pneumophila & Lens & 1 & CIP 108286 \\
\hline L. pneumophila & pneumophila & Paris & 1 & CIP 107629 \\
\hline L. pneumophila & pneumophila & Togus- 1 & 2 & ATCC 33154 \\
\hline L. pneumophila & pneumophila & Bloomington-2 & 3 & ATCC 33155 \\
\hline L. pneumophila & fraseri & Los Angeles-1 & 4 & ATCC 33156 \\
\hline L. pneumophila & pneumophila & Portland 1 & 4 & LTC-14 \\
\hline L. pneumophila & pneumophila & San Francisco & 4 & LTC-15 \\
\hline L. pneumophila & pascuelli & MICU B & 5 & ATCC 33735 \\
\hline L. pneumophila & pascuelli & U8W & 5 & ATCC 33737 \\
\hline L. pneumophila & pneumophila & Cambridge- 2 & 5 & NCTC 11417 \\
\hline L. pneumophila & & Reims & 5 & ATCC 700710 \\
\hline L. pneumophila & fraseri & Dallas 1E & 5 & ATCC 33216 \\
\hline L. pneumophila & pneumophila & Chicago 2 & 6 & ATCC 33215 \\
\hline L. pneumophila & pneumophila & Chicago 8 & 7 & ATCC 33823 \\
\hline L. pneumophila & pneumophila & Concord 3 & 8 & ATCC 35096 \\
\hline L. pneumophila & pneumophila & IN-23-G1-C2 & 9 & ATCC 35289 \\
\hline L. pneumophila & pneumophila & Leiden 1 & 10 & ATCC 43283 \\
\hline L. pneumophila & pneumophila & 797-PA-H & 11 & ATCC 43130 \\
\hline L. pneumophila & pneumophila & $570-\mathrm{CO}-\mathrm{H}$ & 12 & ATCC 43290 \\
\hline L. pneumophila & pneumophila & $82 \mathrm{~A} 3105$ & 13 & ATCC 43736 \\
\hline L. pneumophila & pneumophila & $169-\mathrm{MN}-\mathrm{H}$ & 14 & ATCC 43703 \\
\hline L. pneumophila & fraseri & Lansing 3 & 15 & ATCC 35251 \\
\hline L. pneumophila & fraseri & & & DSM 7514 \\
\hline L. pneumophila & pascullei & & & DSM 7515 \\
\hline L. pneumophila & pneumophila & & & DSM 7513 \\
\hline L. anisa & & & & ATCC 35291 \\
\hline L. anisa & & & & ATCC 35292 \\
\hline L. anisa & & & & DSM 17627 \\
\hline L. beliardensis & & & & DSM 19152 \\
\hline L. birminghamensis & & & & ATCC 43702 \\
\hline L. birminghamensis & & & & ATCC 700709 \\
\hline L. birminghamensis & & & & DSM 19232 \\
\hline L. bozemanae & & & 1 & ATCC 33217 \\
\hline L. bozemanae & & & 2 & ATCC 35545 \\
\hline L. brunensis & & & & DSM 19236 \\
\hline L. drancourtii & & & & A18532-39 \\
\hline L. dumoffii & & & & ATCC 35850 \\
\hline L. dumoffii & & & & ATCC 33279 \\
\hline L. cherrii & & & & DSM 19213 \\
\hline L. cincinnatiensis & & & & DSM 19233 \\
\hline L. erythra & & & 1 & ATCC 35303 \\
\hline L. erythra & & & 2 & ATCC 11987 \\
\hline L. erythra & & & & DSM 17644 \\
\hline L. feeleii & & & & LC 878 \\
\hline L. feeleii & & & & ATCC 700514 \\
\hline L. feeleii & & & 1 & ATCC 35072 \\
\hline L. feeleii & & & 2 & ATCC 35849 \\
\hline L. feeleii & & & & DSM 17645 \\
\hline L. gormanii & & & & ATCC 33297 \\
\hline L. hackeliae & & & 1 & ATCC 35250 \\
\hline L. hackeliae & & & 2 & ATCC 35999 \\
\hline L. hackeliae & & & & DSM 19214 \\
\hline L. impletisoli & & & & DSM 18493 \\
\hline
\end{tabular}


Table 1. cont.

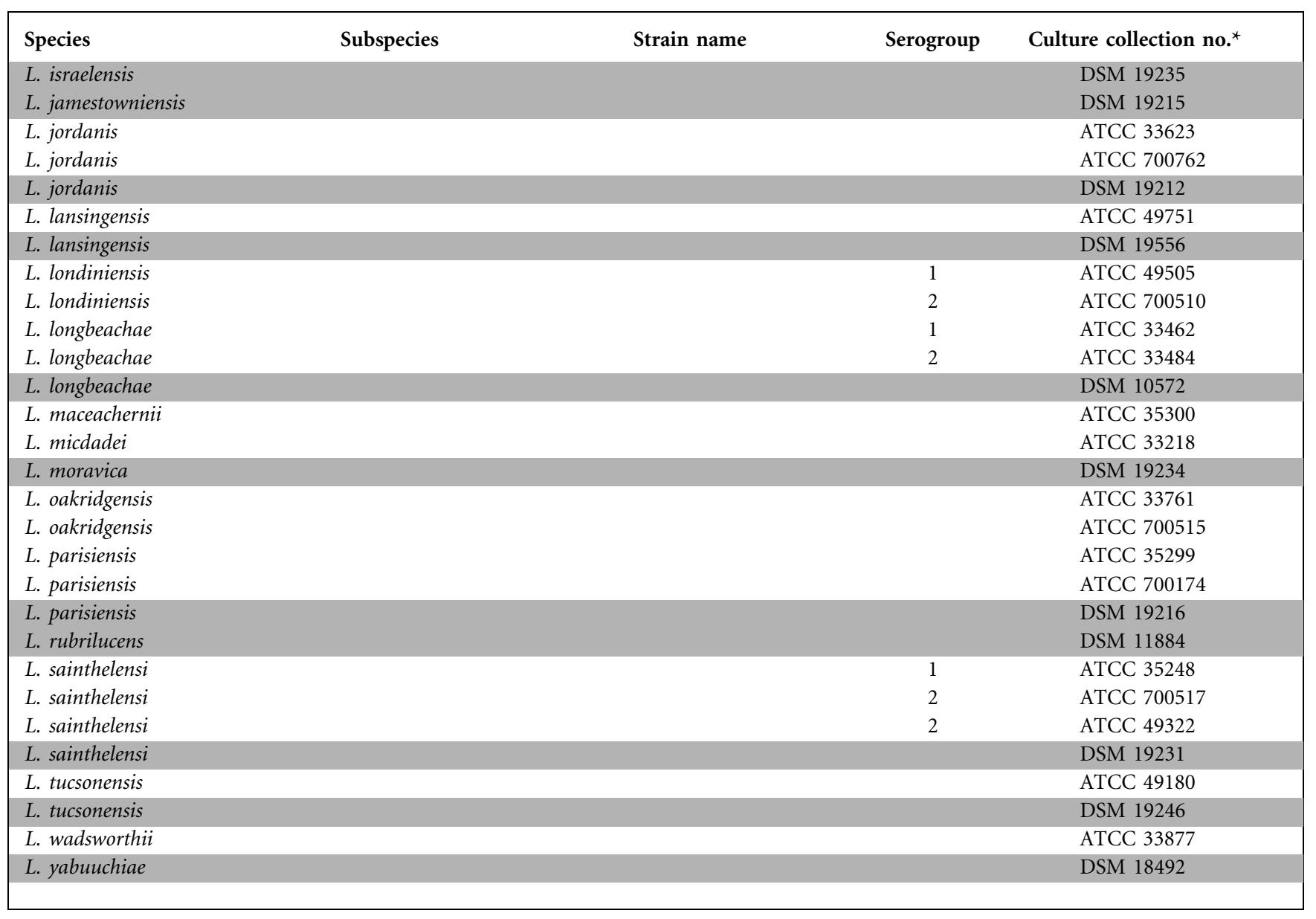

*ATCC, American Type Culture Collection; DSM, Deutsche Sammlung von Mikroorganismen und Zellkulturen GmbH; LC, British Legionella Reference Unit: Respiratory and Systematic Infection Laboratory; LTC, Lyon Type Collection, Centre National de Reference des Legionelles.

amplification and sequencing of the 16S-23S rRNA intergenic spacers or tDNA spacers (De Gheldre et al., 2001; Riffard et al., 1998). Ribotyping and PCR fingerprinting have also demonstrated a high discriminatory power for Legionella species (Salloum et al., 2002; Van Belkum et al., 1993), but may be more adapted to strain genotyping than species identification. However, although reliable, molecular methods are time-consuming and expensive.

Matrix-assisted laser desorption/ionization time-of-flight mass spectrometry (MALDI-TOF-MS) has recently emerged as a rapid and inexpensive bacterial species identification method. It has been shown that most bacterial species exhibit specific protein profiles (Hsieh et al., 2008). MS has been used for efficient discrimination of Arthrobacter species (Vargha et al., 2006), Coxiella burnetii (Skultety et al., 2007), viridans streptococci (Friedrichs et al., 2007), staphylococci (Carbonnelle et al., 2007), Neisseria species (Gudlavalleti et al., 2008; Ilina et al., 2009), Campylobacter species (Kolinska et al., 2008),
Burkholderia cepacia (Miñán et al., 2009; Vanlaere et al., 2008), Clostridium species (Grosse-Herrenthey et al., 2008), Erwinia species (Sauer et al., 2008), Escherichia coli (Parisi et al., 2008; Ruelle et al., 2004), Listeria species (Barbuddhe et al., 2008), non-fermenting bacteria (Degand et al., 2008; Mellmann et al., 2008), oral anaerobic bacteria (Stingu et al., 2008), Yersinia enterocolitica (Parisi et al., 2008), Salmonella species (Dieckmann et al., 2008; Ruelle et al., 2004), Bacteroides species (Nagy et al., 2009) and Bartonella species (Fournier et al., 2009). However, to date, no data are available regarding the identification of Legionella species using MALDI-TOF-MS. In the Bruker database (Bruker Daltonics, Bremen, Germany), containing MALDI-TOF-MS profiles of various bacterial species, only a few Legionella MS profiles are available, with only one spectrum per species or subspecies for 24 Legionella species (Table 1).

In this study, we performed MS analysis on representative strains of all recognized Legionella species with recognized pathogenicity for humans. We then developed a database 
Table 2. MALDI-TOF-MS identification of 114 blind-tested L. pneumophila strains

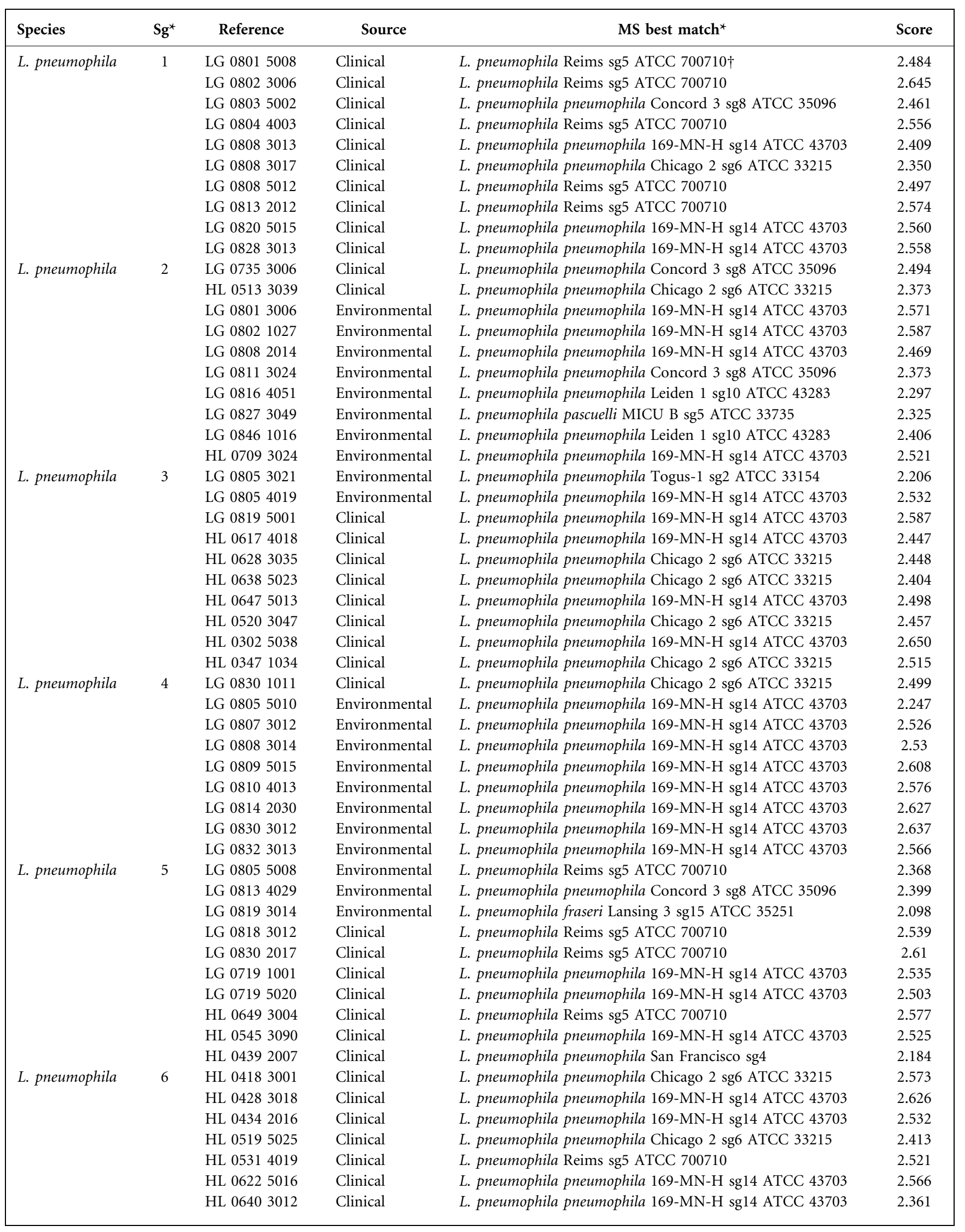


Table 2. cont.

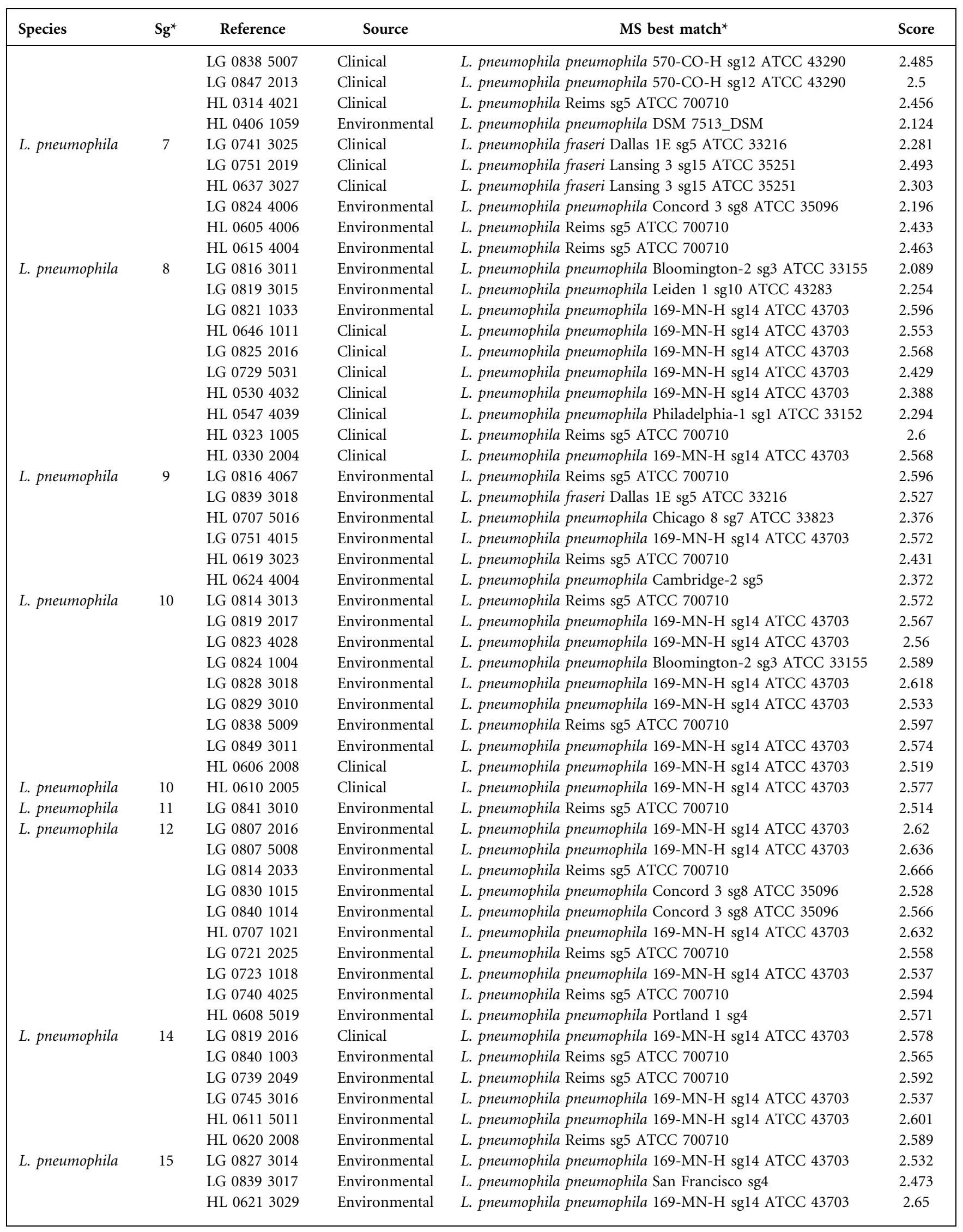


Table 2. cont.

\begin{tabular}{|cccccc|}
\hline Species & Sg $^{*}$ & Reference & Source & MS best match & Score \\
\hline & HL 05053004 & Environmental & L. pneumophila Reims sg5 ATCC 700710 & 2.31 \\
& HL 0505 3005 & Environmental & L. pneumophila Reims sg5 ATCC 700710 & 2.649 \\
\hline
\end{tabular}

${ }^{\star}$ Sg, Serogroup.

$\dagger$ Strain used to evaluate reproducibility.

with the generated spectra, which we tested using a large collection of both clinical and environmental Legionella strains.

\section{METHODS}

Legionella strains. Representative strains of 21 recognized and pathogenic Legionella species were included in our study (including 12 species already referenced in the Bruker database). For some species, several serogroups were evaluated: two for $L$. bozemanae, $L$. erythra, L. feeleii, L. hackeliae, L. londiniensis, L. longbeachae and $L$. sainthelensi, and 15 for L. pneumophila. For each species and serogroup, one or several type strains were used. The 59 Legionella type strains studied are detailed in Table 1 . To evaluate the sensitivity and positive predictive value of MS for the identification of Legionella strains, 237 additional Legionella strains from 19 species were blind tested by MS. These included 114 L. pneumophila (Table 2), seven $L$. anisa, five L. birminghamensis, 11 L. bozemanae, one L. cincinnatiensis, 10 L. dumoffii, 14 L. erythra, seven L. feeleii, seven L. gormanii, six L. jordanis, 12 L. londiniensis, six L. longbeachae, four L. maceachernii, 10 L. micdadei, five $L$. oakridgensis, eight $L$. parisiensis, five L. sainthelensi, four L. tucsonensis and one L. wadsworthii (Table 3). All strains were cultured on buffered charcoal yeast extract $\alpha$-ketoglutarate agar (Oxoid) and incubated for $3-5$ days at $35{ }^{\circ} \mathrm{C}$ in $2.5 \% \mathrm{CO}_{2}$. Species identification of all studied strains was confirmed using mip gene amplification and sequencing as described previously (Ratcliff et al., 1998). The resulting consensus sequences were compared with those in GenBank.

MALDI-TOF-MS. For each strain tested, four isolated colonies were deposited on an MTP 384 target polished steel plate (Bruker Daltonics) and allowed to dry at room temperature. Two microlitres of a matrix solution composed of $\alpha$-cyano-4-hydroxycinnamic acid (Sigma) saturated with $2.5 \%$ trifluoroacetic acid and $50 \%$ acetonitrile was added and allowed to co-crystallize with each sample. Specimens were processed in an Autoflex II MALDI-TOF-MS spectrometer (Bruker Daltonics) using Flex Control software (Bruker Daltonics). Positive ions were extracted with an accelerating voltage of $20 \mathrm{kV}$ in linear mode. Each spectrum indicates the sum of the ions obtained from 675 laser shots performed in nine different regions of the same spot. The spectra were analysed in an $\mathrm{m} / \mathrm{z}$ range of 2000-20 000, and the analysis was performed using Flex Analysis and BioTyper software (Bruker Daltonics). The data obtained from the four replicates were added to minimize random effects. The presence and absence of peaks were considered to be specific profiles for a particular isolate. Only those peaks with a relative intensity above 0.1 that were constantly present in all four sets of data obtained for a given strain were retained. The standard deviation for each conserved peak did not exceed $6 \mathrm{~m} / z$ value. A maximum of 100 peaks with a signal-to-noise ratio of 3 was selected in the range of 3000-15000 Da. From all spectra generated for a single strain, a reference spectrum was determined. A strain was considered to be classified correctly within a species when their reference spectra shared $\geqslant 75$ peaks, with a mass deviation of 200 p.p.m. For the blind test, the 237 tested strains were compared with the complete Bruker database, and with the reference spectra obtained for all 21 Legionella type species tested. Species identification was obtained when a strain demonstrated a best match with a spectrum in the database with a log score $\geqslant 2.0$.

The complete set of spectra obtained in the present study is available in the Unité des Rickettsies Mass Spectrometry (URMS) database online (www.ifr48.com).

Evaluation of the sensitivity and reproducibility of MALDI-TOFMS. Strains correctly identified at the species level, i.e. exhibiting an MS identification identical to their mip identification with a log score $\geqslant 2.0$, were considered true positives, whereas all other strains were considered false negatives. Differences were evaluated using the Mantell-Haenszel test, and were considered significant when $P<0.05$. To evaluate the reproducibility of MALDI-TOF-MS, we retested one strain per species using the same protocol as described above (Tables 2 and 3 ).

\section{RESULTS}

\section{Study of type strains}

Using MALDI-TOF-MS, we obtained protein fingerprints for all 59 reference Legionella strains. Although all tested strains exhibited distinct protein profiles at the species level (Fig. 1), no difference was identified among serogroups within species (Figs 1 and 2). When comparing the spectra obtained from the 59 representative strains with those of 2843 bacteria within the Bruker database, including one spectrum from each of 21 Legionella species, we observed that 39 strains were correctly identified at the species level with a score of $\geqslant 2.0$, and seven strains were correctly identified but with a best score of $<2.0$ against Legionella species. The remaining 13 strains, from nine species, being absent from the Bruker database, obtained a score of $<1.5$ against all bacterial species. All spectra obtained from the 59 type strains were added to our MALDI-TOF-MS database.

\section{Blind test}

When compared with the complete database, spectra obtained from the $114 \mathrm{~L}$. pneumophila strains enabled unambiguous identification at the species level with $\log$ scores of $\geqslant 2.0$ against $L$. pneumophila only. However, scores of $\geqslant 2.0$ were obtained for all strains against all $L$. pneumophila serogroups, thus demonstrating that MALDITOF-MS is not discriminatory at the serogroup level 
Table 3. MALDI-TOF-MS identification of 123 blind-tested strains from various pathogenic Legionella species

Strains correctly identified at the species level but with scores $<2.0$ are shaded in pale grey; strains misidentified at the species level with scores $<2.0$ are shaded in dark grey; strains misidentified at the species level with scores $\geqslant 2.0$ are shaded in black.

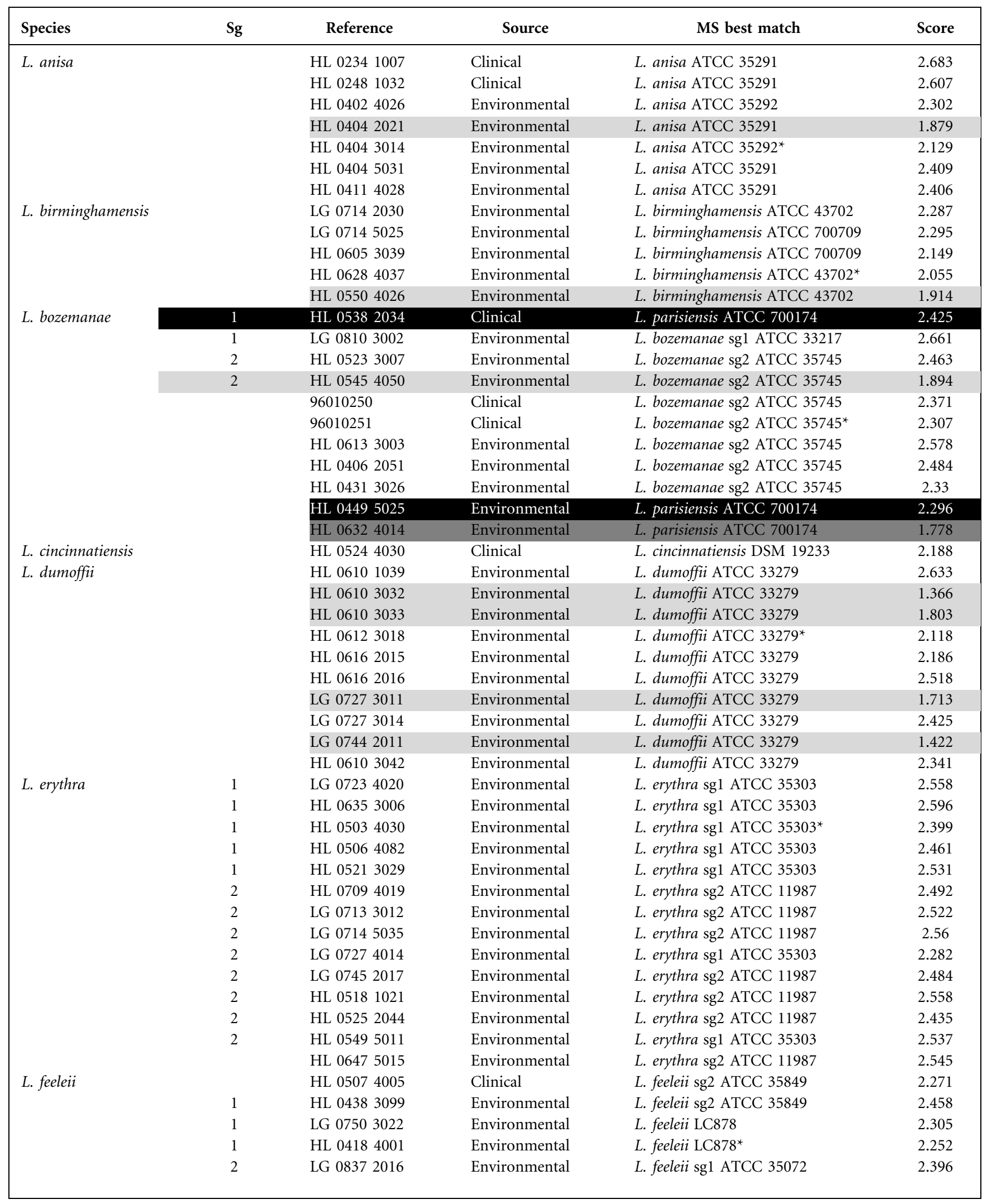


Table 3. cont.

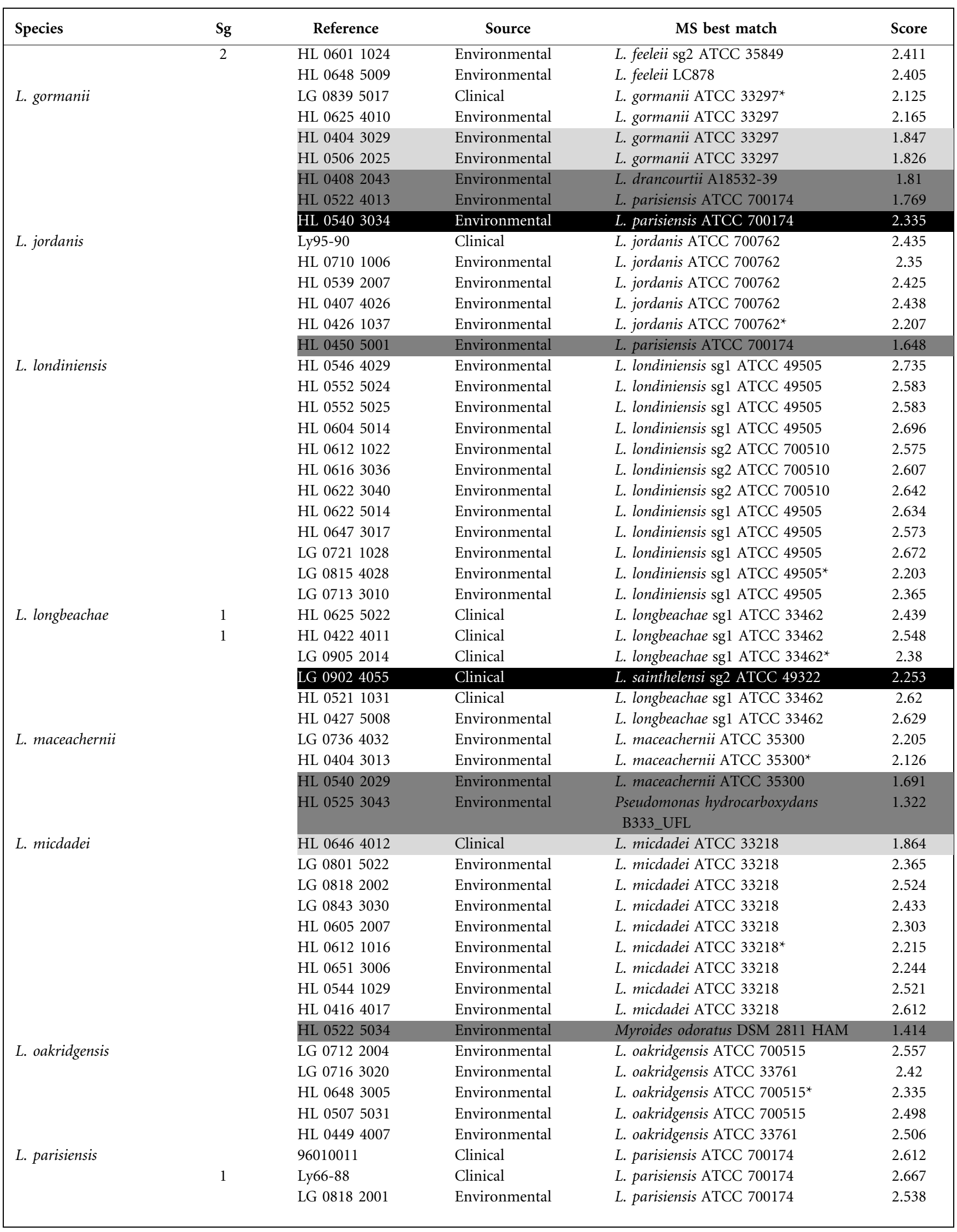


Table 3. cont.

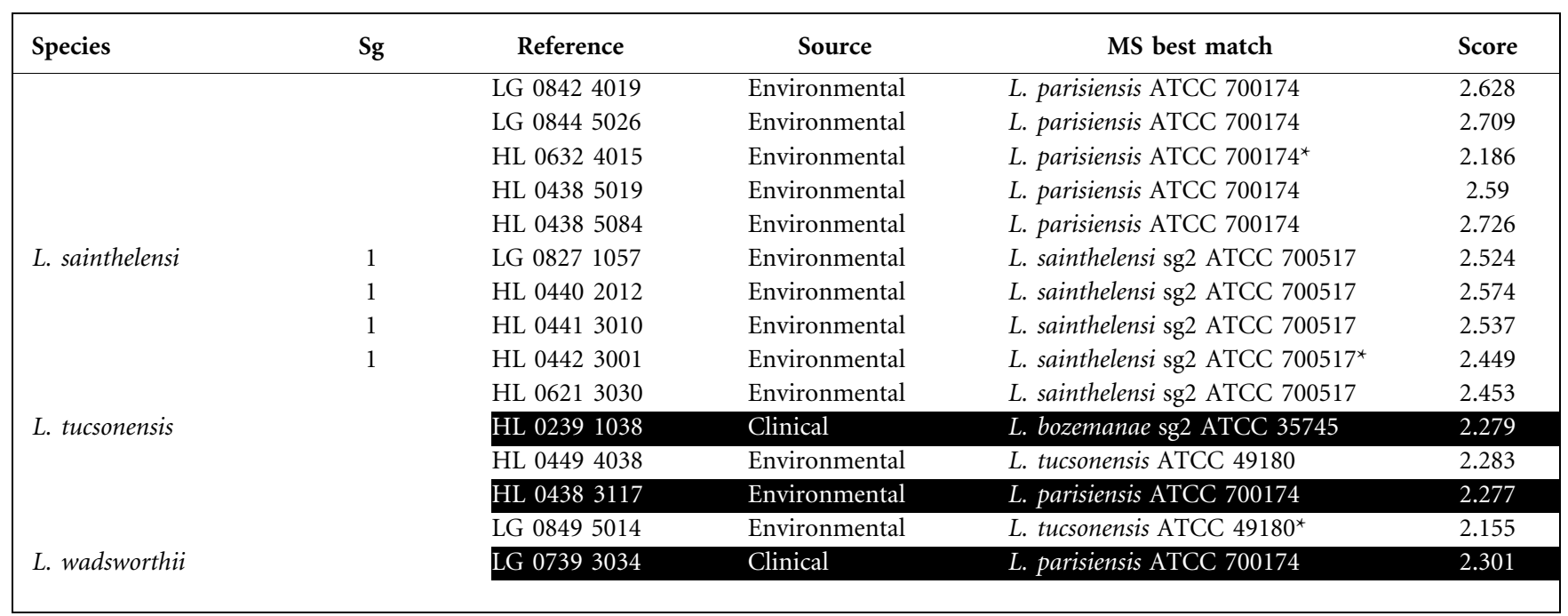

*Strains used to evaluate reproducibility.

(Table 2). Among the other 123 blind-tested strains (Table 3), 99 were identified correctly at the species level with a score of $\geqslant 2.0(80.5 \%) ; 17(13.8 \%)$ exhibited a score of
$<2.0$, comprising 10 against their homologous species, five against an heterologous Legionella species and two against a non-Legionella bacterial species; and seven strains exhibited

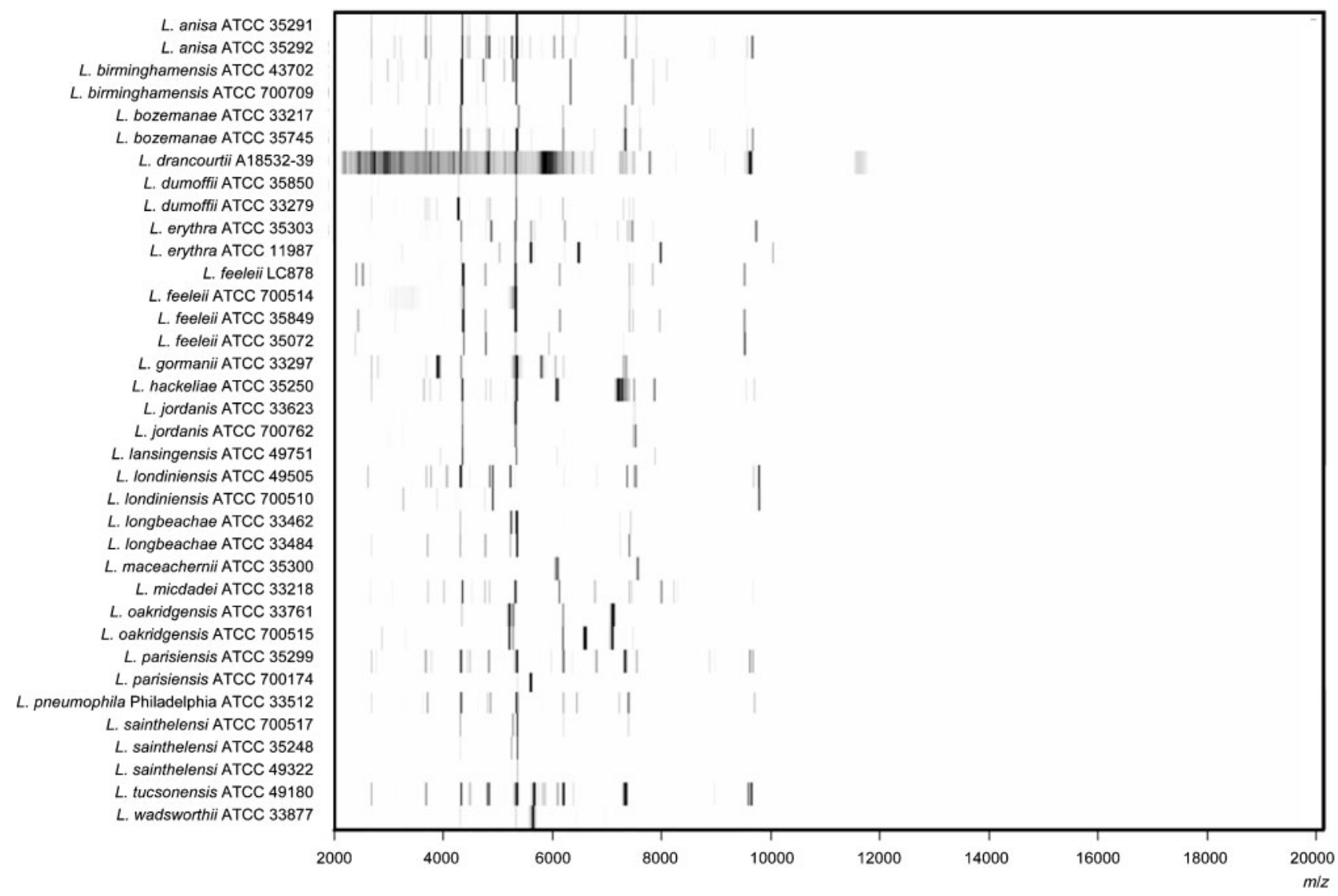

Fig. 1. Virtual gel view of MS reference spectra from the tested Legionella species. 


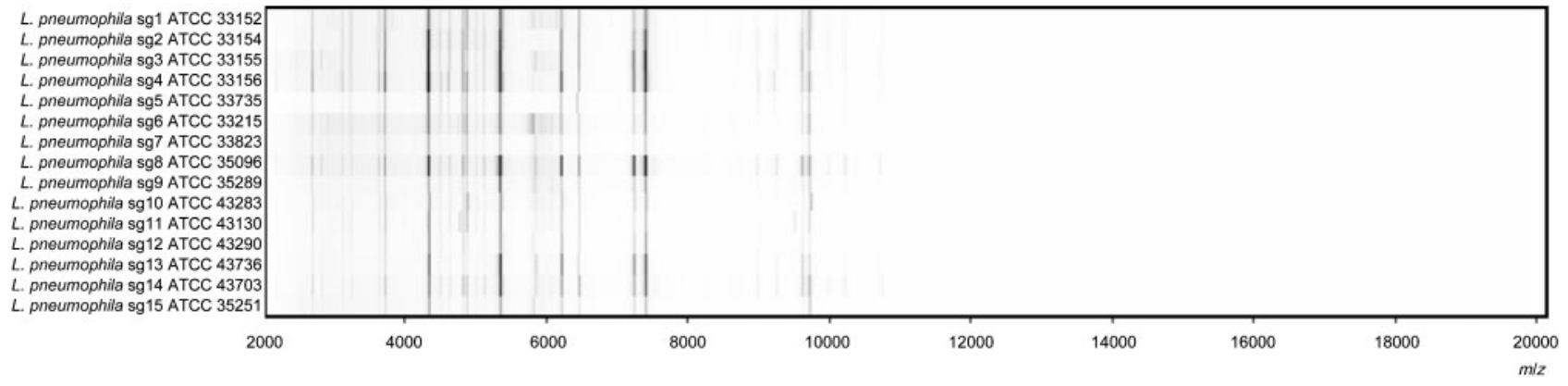

Fig. 2. Virtual gel view of MS reference spectra from the tested Legionella pneumophila serogroups.

a score of $\geqslant 2.0$ against a heterologous Legionella species. As with L. pneumophila, spectra obtained from the different serogroups of other species exhibited a score of $\geqslant 2.0$ against all serogroups of their homologous species.

Thus, the overall sensitivity of MALDI-TOF-MS for the identification of Legionella strains was $99.2 \%$ at the genus level $(235 / 237)$ and $89.9 \%$ at the species level (213/237). In addition, strains with a score of $<2.0$ were correctly identified at the species level in $58.8 \%$ of cases. However, the sensitivity varied among species. There was $100 \%$ sensitivity in eight species: L. pneumophila (114/114), L. cincinnatiensis (1/1), L. erythra (14/14), L. feeleii (7/7), L. londiniensis (12/12), L. oakridgensis (5/5), L. parisiensis (8/ 8 ) and L. sainthelensi (5/5). The sensitivity was lower in the other 11 tested species: $85.7 \%$ in L. anisa (6/7), 83.3\% (5/ 6 ) in L. jordanis and L. longbeachae, $80.0 \%(4 / 5)$ in $L$. birminghamensis and L. micdadei, $63.6 \%$ in L. bozemanae (7/11), $60.0 \%$ in L. dumoffii $(6 / 10), 50.0 \%(2 / 4)$ in $L$. maceachernii and L. tucsonensis, $28.6 \%$ in L. gormanii $(2 / 7)$ and $0 \%$ in L. wadsworthii $(0 / 1)$. In the reproducibility assay, the 19 strains tested obtained the same species identifications as in the blind test, with scores of $>2.0$ for all strains. For seven out of eight of the species with $100 \%$ of strains correctly identified, our database included more than two reference spectra, which was the case in only four of the 11 remaining species $(P=0.03)$.

\section{DISCUSSION}

The ability of various Legionella species to cause pneumonia and their potential role in nosocomial infections has stimulated the development of efficient identification tools, enabling rapid and adequate management of patients. However, precise identification techniques commonly used for Legionella species require molecular methods that are expensive and time-consuming. Here, we used MALDITOF-MS, a rapid and cost-effective identification method, for the identification of Legionella at the species level. Obtaining a strain identification took only $10 \mathrm{~min}$ from selecting a colony grown on the agar plate, with up to 15 strains being analysed per hour at a total cost of $€ 1.65$ per strain (including personnel cost).
We demonstrated that the discriminatory power of this method for identifying Legionella strains at the species level was high. We first developed a spectrum database using 59 representative strains from 21 species, and observed that all species could be differentiated (Figs 1 and 2), although some species, such as L. longbeachae and L. sainthelensi, exhibited closely related protein spectra. Next, we tested the validity of this method by performing a blind test with 237 strains from 19 different species. Among these, we observed that the discriminatory power of MALDI-TOFMS varied according to the tested species, with strains from eight species being correctly identified in $100 \%$ of cases, whereas, for the remaining 11 species, the degree of proper identification varied from 0 to $85.7 \%$. Such differences might be explained by an intraspecific variability of protein profiles among strains. We observed that Legionella strains were more likely to be correctly identified at the species level when more than two reference spectra were present in the database $(P=0.03)$ (Table 1$)$. In addition, for seven strains, a score of $>2.0$ was obtained against a species that differed from but was phylogenetically close to their homologous species (Adeleke et al., 1996; Grattard et al., 2006; Ko et al., 2002). These results suggest that the discriminatory power of MALDI-TOF-MS may be improved by increasing the number of spectra per species in the database. All spectra obtained in this study were added to our database (www.ifr48.com).

According to our results, we estimated the sensitivity of MALDI-TOF-MS for Legionella isolate identification to be 99.2 and $89.9 \%$ at the genus and species level, respectively (Tables 2 and 3). However, although we demonstrated that MALDI-TOF-MS is a powerful tool for the identification of Legionella strains at the species level, notably, for the most common pathogenic species, L. pneumophila, it could not discriminate among serogroups. In Listeria species, MALDI-TOF has previously been demonstrated to be as discriminatory as PFGE at the serogroup level (Barbuddhe et al., 2008). However, for Legionella species, reference spectra from the different serogroups enabled correct species identification, but were too similar to be discriminatory at the serogroup level. Antigenic variations among Legionella serogroups have mainly been linked to differ- 
ences in the LPS and major outer-membrane protein (Benson \& Fields, 1998). Our data suggest that these protein differences are not easily detectable by MALDITOF-MS. However, we cannot rule out the possibility that these results were due to our mode of preparation of bacteria.

In conclusion, we determined reference MS spectra for cultivated and recognized pathogenic Legionella species. We demonstrated that species identification by MALDITOF-MS is reproducible and relevant for the genus Legionella and presents several advantages over other identification tools, including speed, discriminatory power and low cost. We added to the existing URMS database all spectra generated from this study, spanning 21 different pathogenic species. For Legionella strains showing intraspecific protein profile variability, this database will continuously be supplemented with spectra from additional strains to increase the representativity of species.

\section{REFERENCES}

Adeleke, A., Pruckler, J., Benson, R., Rowbotham, T., Halablab, M. \& Fields, B. (1996). Legionella-like amebal pathogens - phylogenetic status and possible role in respiratory disease. Emerg Infect Dis 2, 225-230.

Barbuddhe, S. B., Maier, T., Schwarz, G., Kostrzewa, M., Hof, H., Domann, E., Chakraborty, T. \& Hain, T. (2008). Rapid identification and typing of Listeria species by matrix-assisted laser desorption ionization-time of flight mass spectrometry. Appl Environ Microbiol 74, 5402-5407.

Benson, R. F. \& Fields, B. S. (1998). Classification of the genus Legionella. Semin Respir Infect 13, 90-99.

Carbonnelle, E., Beretti, J. L., Cottyn, S., Quesne, G., Berche, P., Nassif, X. \& Ferroni, A. (2007). Rapid identification of staphylococci isolated in clinical microbiology laboratories by matrix-assisted laser desorption ionization-time of flight mass spectrometry. J Clin Microbiol 45, 2156-2161.

Degand, N., Carbonnelle, E., Dauphin, B., Beretti, J. L., Le Bourgeois, M., Sermet-Gaudelus, I., Segonds, C., Berche, P., Nassif, X. \& Ferroni, A. (2008). Matrix-assisted laser desorption ionization-time of flight mass spectrometry for identification of nonfermenting Gram-negative bacilli isolated from cystic fibrosis patients. J Clin Microbiol 46, 3361-3367.

De Gheldre, Y., Maes, N., Presti, F. L., Etienne, J. \& Struelens, M. (2001). Rapid identification of clinically relevant Legionella spp. by analysis of transfer DNA intergenic spacer length polymorphism. J Clin Microbiol 39, 162-169.

Dieckmann, R., Helmuth, R., Erhard, M. \& Malorny, B. (2008). Rapid classification and identification of salmonellae at the species and subspecies levels by whole-cell matrix-assisted laser desorption ionization-time of flight mass spectrometry. Appl Environ Microbiol 74, 7767-7778.

Fournier, P. E., Couderc, C., Buffet, S., Flaudrops, C. \& Raoult, D. (2009). Rapid and cost-effective identification of Bartonella species using mass spectrometry. J Med Microbiol 58, 1154-1159.

Friedrichs, C., Rodloff, A. C., Chhatwal, G. S., Schellenberger, W. \& Eschrich, K. (2007). Rapid identification of viridans streptococci by mass spectrometric discrimination. J Clin Microbiol 45, 2392-2397.

Garrity, G. M., Bell, J. A. \& Lilburn, T. (2005). Order VI. Legionellales ord. nov. In Bergey's Manual of Systematic Bacteriology, pp. 210-236.
Edited by D. J. Brenner, N. R. Krieg, J. J. Staley \& G. M. Garrity. New York: Springer.

Grattard, F., Ginevra, C., Riffard, S., Ros, A., Jarraud, S., Etienne, J. \& Pozzetto, B. (2006). Analysis of the genetic diversity of Legionella by sequencing the 23S-5S ribosomal intergenic spacer region: from phylogeny to direct identification of isolates at the species level from clinical specimens. Microbes Infect 8, 73-83.

Grosse-Herrenthey, A., Maier, T., Gessler, F., Schaumann, R., Bohnel, H., Kostrzewa, M. \& Kruger, M. (2008). Challenging the problem of clostridial identification with matrix-assisted laser desorption and ionization-time-of-flight mass spectrometry (MALDI-TOF MS). Anaerobe 14, 242-249.

Gudlavalleti, S. K., Sundaram, A. K., Razumovski, J. \& Doroshenko, V. (2008). Application of atmospheric pressure matrix-assisted laser desorption/ionization mass spectrometry for rapid identification of Neisseria species. J Biomol Tech 19, 200-204.

Hacker, J. \& Fischer, G. (1993). Immunophilins: structure-function relationship and possible role in microbial pathogenicity. $\mathrm{Mol}$ Microbiol 10, 445-456.

Hsieh, S. Y., Tseng, C. L., Lee, Y. S., Kuo, A. J., Sun, C. F., Lin, Y. H. \& Chen, J. K. (2008). Highly efficient classification and identification of human pathogenic bacteria by MALDI-TOF MS. Mol Cell Proteomics 7, 448-456.

Ilina, E. N., Borovskaya, A. D., Malakhova, M. M., Vereshchagin, V. A., Kubanova, A. A., Kruglov, A. N., Svistunova, T. S., Gazarian, A. O., Maier, T. \& other authors (2009). Direct bacterial profiling by matrixassisted laser desorption-ionization time-of-flight mass spectrometry for identification of pathogenic Neisseria. J Mol Diagn 11, 75-86.

Ko, K. S., Lee, H. K., Park, M. Y., Lee, K. H., Yun, Y. J., Woo, S. Y., Miyamoto, H. \& Kook, Y. H. (2002). Application of RNA polymerase $\beta$-subunit gene $(r p o B)$ sequences for the molecular differentiation of Legionella species. J Clin Microbiol 40, 2653-2658.

Kolinska, R., Drevinek, M., Jakubu, V. \& Zemlickova, H. (2008). Species identification of Campylobacter jejuni ssp. jejuni and C. coli by matrix-assisted laser desorption/ionization time-of-flight mass spectrometry and PCR. Folia Microbiol (Praha) 53, 403-409.

Mellmann, A., Cloud, J., Maier, T., Keckevoet, U., Ramminger, I., Iwen, P., Dunn, J., Hall, G., Wilson, D. \& other authors (2008). Evaluation of matrix-assisted laser desorption ionization-time-offlight mass spectrometry in comparison to $16 \mathrm{~S}$ rRNA gene sequencing for species identification of nonfermenting bacteria. J Clin Microbiol 46, 1946-1954.

Miñán, A., Bosch, A., Lasch, P., Stämmler, M., Serra, D. O., Degrossi, J., Gatti, B., Vay, C., Daquino, M. \& other authors (2009). Rapid identification of Burkholderia cepacia complex species including strains of the novel Taxon $\mathrm{K}$, recovered from cystic fibrosis patients by intact cell MALDI-ToF mass spectrometry. Analyst 134, 1138-1148.

Nagy, E., Maier, T., Urban, E., Terhes, G., Kostrzewa, M. \& ESCMID Study Group on Antimicrobial Resistance in Anaerobic Bacteria (2009). Species identification of clinical isolates of Bacteroides by matrix-assisted laser-desorption/ionization time-of-flight mass spectrometry. Clin Microbiol Infect 15, 796-802.

Parisi, D., Magliulo, M., Nanni, P., Casale, M., Forina, M. \& Roda, A. (2008). Analysis and classification of bacteria by matrix-assisted laser desorption/ionization time-of-flight mass spectrometry and a chemometric approach. Anal Bioanal Chem 391, 2127-2134.

Ratcliff, R. M., Lanser, J. A., Manning, P. A. \& Heuzenroeder, M. W. (1998). Sequence-based classification scheme for the genus Legionella targeting the mip gene. J Clin Microbiol 36, 1560-1567.

Riffard, S., Lo, P. F., Normand, P., Forey, F., Reyrolle, M., Etienne, J. \& Vandenesch, F. (1998). Species identification of Legionella via 
intergenic 16S-23S ribosomal spacer PCR analysis. Int J Syst Bacteriol 48, 723-730.

Ruelle, V., El, M. B., Zorzi, W., Ledent, P. \& Pauw, E. D. (2004). Rapid identification of environmental bacterial strains by matrix-assisted laser desorption/ionization time-of-flight mass spectrometry. Rapid Commun Mass Spectrom 18, 2013-2019.

Salloum, G., Meugnier, H., Reyrolle, M., Grimont, F., Grimont, P. A., Etienne, J. \& Freney, J. (2002). Identification of Legionella species by ribotyping and other molecular methods. Res Microbiol 153, 679-686.

Sauer, S., Freiwald, A., Maier, T., Kube, M., Reinhardt, R., Kostrzewa, M. \& Geider, K. (2008). Classification and identification of bacteria by mass spectrometry and computational analysis. PLoS One 3, e2843.

Skultety, L., Hernychova, L., Bereghazyova, E., Slaba, K. \& Toman, R. (2007). Detection of specific spectral markers of Coxiella burnetii isolates by MALDI-TOF mass spectrometry. Acta Virol 51, 55-58.
Stingu, C. S., Rodloff, A. C., Jentsch, H., Schaumann, R. \& Eschrich, K. (2008). Rapid identification of oral anaerobic bacteria cultivated from subgingival biofilm by MALDI-TOF-MS. Oral Microbiol Immunol 23, 372-376.

Van Belkum, A., Struelens, M. \& Quint, W. (1993). Typing of Legionella pneumophila strains by polymerase chain reactionmediated DNA fingerprinting. J Clin Microbiol 31, 2198-2200.

Vanlaere, E., Sergeant, K., Dawyndt, P., Kallow, W., Erhard, M., Sutton, H., Dare, D., Devreese, B., Samyn, B. \& Vandamme, P. (2008). Matrix-assisted laser desorption ionisation-time-of of-flight mass spectrometry of intact cells allows rapid identification of Burkholderia cepacia complex. J Microbiol Methods 75, 279-286.

Vargha, M., Takats, Z., Konopka, A. \& Nakatsu, C. H. (2006). Optimization of MALDI-TOF MS for strain level differentiation of Arthrobacter isolates. J Microbiol Methods 66, 399-409. 